\title{
A MECHANISM FOR CIRCULAR POLARIZATION IN PULSAR RADIATION
}

\author{
A. Z. Kazbegi, G. Z. Machabeli and G. I. Melikidze \\ Abastumani Astrophysical Observatory
}

Observational data show that many pulsars exhibit a moderate amount of circular polarization (CP) as well as linearly polarized emission. Generally, the degree of $\mathrm{CP}$ is of the order of several percent, but some of pulsars have higher $\mathrm{CP}-$ about 20\% (Manchester and Taylor 1977, Taylor and Stinebring 1986) and PSR 1702-19 has 60\% (Biggs et al. 1988). No satisfactory explanation for the existence of this CP is now known, and we propose in this paper to outline a possible mechanism for the generation of this CP consistent with our theory of pulsar radio-wave excitation. In Kazbegi et al. (1992; hereafter Paper I) we mentioned that the distribution functions of electrons $F^{-}$and positrons $F^{+}$are shifted with respect to each other due to the presence of the primary electron beam and the quasi-neutrality of the plasma as a. whole. The value

$$
\Delta \gamma=\gamma_{+}-\gamma_{-}=\int F^{+} \gamma d p-\int F^{-} \gamma d p
$$

where $\int F^{ \pm} d p=1$, determines the difference in energies of electrons and positrons. The value of $\Delta \gamma$ is small but it has decisive significance in explaining the polarization properties of the emission.

Let us investigate the problem of the polarization of waves propagating in the magnetospheric plasma. From eq.(1) of Paper I, it follows that the curvature of field lines is not important for the form of $\epsilon_{i j}^{p}$. Thus the Cartesian coordinate system can be used. We shall be interested only in the waves propagating in the observer's direction. Because of relativistic compression the angle between the local magnetic field $B$ and wave vector $k$ is small, $\theta^{2}=\left(k_{\perp} / k_{z}\right)^{2} \ll 1$. For $\theta^{2} \ll 1$, $\Delta \gamma / \gamma \ll 1$ and $\omega_{\mathrm{p}}^{2} / \omega_{\mathrm{B}}^{2} \ll 1$, one can obtain from Shafranov (1963) that

$$
\text { if } \quad \theta^{2} \gg\left(\frac{\omega}{8 \omega_{\mathrm{B}}}\right) \frac{\Delta \gamma}{\gamma_{\mathrm{p}}^{4}} ;
$$

(where $\gamma_{p}$ is the average Lorentz factor of the bulk of plasma) then two linearly polarized waves in the relativistic electron-positron plasma can exist, $E_{x} / E_{y} \ll 1$ or $E_{x} / E_{y} \gg 1$. The first mode has the prevailing component $E_{y}$, i.e. the electric field vector $E$ lies in the plane of $\boldsymbol{k}$ and $B$. This is a mixed electrostatic-electromagnetic lt-wave. The electric vector $E_{x}$ of the second wave is normal to the $B$ - $k$ plane. This electromagnetic wave is called the transverse $\mathrm{t}$-wave.

In the opposite case, when

$$
\theta^{2} \ll(\omega \Delta \gamma) /\left(8 \omega_{\mathrm{B}} \gamma_{\mathrm{p}}^{4}\right)
$$

one obtains two CP waves with left-hand (in the direction of electron rotation) and right-hand (in the direction of positron rotation) polarization. The intermediate cases have elliptical polarization since generally $E=E_{x}+i E_{y}$. In what follows we will discuss only waves with $\theta^{2} \ll 1$ and $k_{\perp} v_{\perp} / \omega_{\mathrm{B}} \ll 1$ where $v_{\perp}=p_{\perp} c / \gamma$.

For energetic particles only the resonance at the anomalous Doppler-effect (Paper I) can be satisfied. Therefore for the resonant part of the $\epsilon_{i j}$ one obtains the following dispersion relation for $t$-waves

$$
\frac{k^{2} c^{2}}{\omega^{2}}=\epsilon_{x x}^{\mathrm{p}} \pm i \epsilon_{r x}^{\mathrm{p}}-\left(\epsilon_{1}^{ \pm}+\epsilon_{2}^{ \pm}\right)
$$

where

$$
\epsilon_{1}^{ \pm}+\epsilon_{2}^{ \pm}=\frac{\omega_{\text {pres }}^{2}}{\omega^{2}} \int \frac{f^{ \pm}\left(\omega-k_{\varphi} v_{\varphi}-\frac{k_{\varphi} u^{2}}{c} \mp \frac{k_{x} u}{2}\right) d p_{\varphi}}{\gamma\left(\omega-k_{\varphi} v_{\varphi} \mp k_{x} u+\omega_{\mathrm{B}} / \gamma\right)}
$$

The growth rate of the cyclotron instability is given in Paper I, eqs.(7) and (8).

Below we demonstrate that the main polarization features observed in pulsar radiation can be explained within the framework of our theory without any additional assumptions. The condition of eq.(2) is fulfilled better when the impact parameter is larger-i.e., for larger $\theta$. In this case the emission should be characterized by the two linearly polarized $\mathrm{t}$ and lt-modes $\left(\boldsymbol{E}_{\mathrm{t}} \cdot \boldsymbol{E}_{\mathrm{lt}}\right)=0$ (Kazbegi et al. 1988). The transitions between the two modes are described elsewhere (Kazbegi, Machabeli, and Melikidze 1989). According to this paper the transitions are accompanied by changes of dominance between "core" and "cone"-type emissions. We believe that our two orthogonal plasma modes can be identified with the orthogonal modes observed in pulsar radiation (Stinebring et al. 1984a).

If $\Delta \gamma$ is so small that the inequality eq.(2) is fulfilled even for small angles $\left(\theta^{2} \ll 1\right)$, we have only linearly polarized modes. The electric vector of the $t$-waves is directed normal to the plane of $\boldsymbol{k}$ and $\boldsymbol{B}$, whereas $\boldsymbol{E}_{1 \mathfrak{t}}$ lies in the $\boldsymbol{k}-\boldsymbol{B}$ plane. For minimal values of $k_{\perp}$ (in the center of the pulse window) $k$ is almost parallel to $B$. The $k-B$ plane 
then degenerates into a line. Consequently one cannot distinguish between the $t$ and lt-modes since the orientation of both $\boldsymbol{E}_{\mathrm{t}}$ and $\boldsymbol{E}_{\mathrm{lt}}$ is arbitrary. The observed emission is then unpolarized. Single pulses often demonstrate a minimum of linear polarization at their intensity maximum e.g., (Ferguson and Seiradakis 1978).

If the sight line cuts the cone of "core"-type emission near the center, then in the midpoint of the pulse window the waves having small $\theta$ (in our model the smallness of $\theta$ is identical with the smallness of the impact parameter) are observed. In this case there is a high probability for the condition of eq.(3) to be fulfilled. Then $E_{x} / E_{y}= \pm 1$, and there are two waves. Eqs.(7) and (8) in Paper I lead to the possibility of wave generation involving both electrons and positrons. Electrons produce the left-hand $(+)$ and positrons the right-hand $(-)$ polarization. However the waves are excited only if the following resonance condition is satisfied

$$
\delta=\frac{\omega_{\mathrm{B}}}{k_{\varphi} c \gamma_{\mathrm{res}}}+\frac{1}{2}\left(\frac{k_{x}}{k_{\varphi}}-\frac{u_{\alpha}}{c}\right)^{2}+\frac{1}{2} \frac{k_{r}^{2}}{k_{\varphi}^{2}}
$$

Eq.(5) can be met in two cases: First, when

$$
2 \delta>\max \left\{\left(\frac{k_{\perp}}{k_{\varphi}}\right)^{2} ;\left(\frac{u}{c}\right)^{2}\right\},
$$

that is, for both species of particles that excite the waves with the growth rate given by eq.(7) in Paper I. Second, when

$$
\frac{k_{r}}{k_{\varphi}} \ll 2 \delta \leq\left\{\left(\frac{k_{x}}{k_{\varphi}}\right)^{2} ;\left(\frac{u}{c}\right)^{2}\right\},
$$

with the growth rate given by eq.(8) in Paper I. The drift velocity $u$ is directed perpendicular to the plane of curvature of field lines. To satisfy eq. (5) the additional strict condition $k_{x} / k_{\varphi}=u_{\alpha} / c$ is necessary.

Let us consider the latter case first. The planes of the open magnetic field lines are locally almost parallel to each other (they have the same radius of curvature). Therefore one can choose the plane of the field lines as the $r \theta \varphi$ co-ordinate plane (see Paper I). In such a case the drift velocity $u$ does not change its orientation, whereas the wave vector $\boldsymbol{k}$ does change as the sight line cuts the emission cone. One observes a succession of orientations of $\boldsymbol{k}$ along the cone. The condition

$$
\left(\frac{k_{x}}{k_{\varphi}}-\frac{u_{\alpha}}{c}\right)^{2} \equiv \frac{k_{x}^{2}}{k_{\varphi}^{2}}+\frac{u^{2}}{c^{2}}-2 \frac{k_{\perp}}{k_{\varphi}} \frac{u_{\alpha}}{c} \cos \psi=0
$$

is fulfilled only if $k_{\perp} u_{\alpha} \cos \psi>0$. The sight line can cut the emission cone in such a way that the angle $\psi$ passes through $\pi / 2$, and the sign of $\cos \psi$ reverses. For the value $k_{\perp} u_{\alpha} \cos \psi$ to remain positive it is necessary to change the sense of the drift velocity (the drift caused by the magnetic field curvature forces electrons and positrons to drift in opposite directions)-i.e. for angles of $-\pi / 2<\psi<\pi / 2$ the resonance condition eq.(5) is fulfilled for positrons (right-hand CP), and for $\pi / 2<\psi<3 \pi / 2$ for electrons (left-hand CP). Hence, a sight line traverse of the emission cone in a place where $\psi$ changes its sense (near $\psi \approx \pi / 2$ ) is accompanied by a change of the sense of the CP. Such reversals are observed, e.g. for PSR $1237+25,1839+09$, $1907+03$ and others (For details see Radhakrishnan and Rankin 1990.). If $\psi \rightarrow \pi / 2, \cos \psi \rightarrow 0$, and the resonance condition of eq. (5) breaks down because $\left(k_{x} / k_{\varphi}-u_{\alpha} / c\right)^{2}>\delta$. Hence when the sight line passes through $\pi / 2$ the $C P$ will decrease.

Let us go back to the case of eq. $(A)$. Both species of particles satisfy the condition expressed in eq.(5). Thus the intensity of the CP emission must be considerably reduced because it is proportional to the difference in number of resonant electrons and positrons. But we have more positrons in the resonant region. Hence waves are excited having right-hand $\mathrm{CP}$ with reduced intensity. If the distribution functions coincide $(\Delta \gamma=0)$, the two modes of $\mathrm{CP}$ superpose giving two linearly polarized waves having electric vectors which are orthogonal to each other. As was shown they can give rise to unpolarized emission in the case when $k_{\perp} \rightarrow 0$.

The spectrum of excited $t$-waves is given by eq. (2) of Paper $I$, and the resonant frequency is $\omega_{0}=\omega_{\mathrm{B}} / \delta \gamma_{\mathrm{res}}$. In a dipole magnetic field one can assume that $B=B_{0}\left(R_{0} / R\right)^{3}$ and $n=n_{0}\left(R_{0} / R\right)^{3}$, where the index ' 0 ' denotes the values taken at the stellar surface. Using these expressions one obtains $\omega_{0}=\operatorname{const}\left(R_{0} / R\right)^{6}$. This relationship suggests the so-called radius-to-frequency mapping phenomenon in which the lower frequencies are generated at larger distances from the star. At the same time $\delta=\delta_{0}\left(R / R_{0}\right)^{3}$ and $(u / c)=\left(u_{0} / c\right)\left(R / R_{0}\right)^{2}$. Comparison of these relations shows that at distances $R<10^{9} \mathrm{~cm}(u / c)^{2}<\delta$, and for $R>10^{9} \mathrm{~cm}$ $\delta<(u / c)^{2}$ (for $P \approx 1 \mathrm{~s}$ ). Thus the probability for the $\mathrm{CP}$ to reverse sense (it occurs when $\delta<(u / c)^{2}$ ) is higher at distances $R>10^{9} \mathrm{~cm}$, and hence at lower frequencies.

Finally we emphasize that within our theory we have the possibility for both sense-reversing and single sense circular polarization. Sense-reversing CP requires significant drift velocity $u$, and single sense CP will occur even for $u=0$, because it is only necessary that $\Delta \gamma>0$ (see Paper I).

Acknowledgments: We would like to thank Dr. J. Gil for helpful discussions and help with preparing the final version of this paper. 